\title{
Perfiles de personalidad y síndromes clínicos en personas sin hogar
}

\author{
José M. Rodríguez-Pellejero, Juan L. Núñez y Débora Hernández \\ Universidad de Las Palmas de Gran Canaria, España
}

Personality profiles and clinical syndromes in homeless

\begin{abstract}
The aims of this study were to examine the presence of clinical personality patterns and clinical syndromes in homeless, and to identify common personality profiles. Participants were 144 subjects, 35 women and 109 men (mean age $=42$ years), all of them in a situation of chronic social exclusion, $67 \%$ were homeless for more than a year, and $33 \%$ more than three years. During the evaluation period they resided in a shelter for the homeless. Results showed that clinical personality patterns and clinical syndromes with higher prevalence, according to the model of Million, were: depressive, narcissistic and paranoid, as well as anxiety, drug dependence and thought disorder, respectively. In addition, cluster analysis classified the subjects into two groups «narcissistic-adaptive» and «depressive-paranoid». These results suggested that the treatment and health resources should be adapted according to the psychiatric deterioration, considering the homeless as a heterogeneous group with different psychosocial needs.
\end{abstract}

Keywords: Homelessness; homeless; personality disorders; personality profile.

Resumen: Los objetivos de este trabajo fueron: analizar la prevalencia de patrones clínicos de personalidad y de síndromes clínicos, examinar la relación entre ambos e identificar perfiles de personalidad comunes en una muestra de personas sin hogar. Los participantes fueron 144 sujetos, 35 mujeres y 109 hombres, con una media de edad de 42 años, todos ellos en una situación de exclusión social crónica, el 67\% llevaba sin hogar un periodo de tiempo superior a un año, y el 33\% más de tres años. Durante el periodo de evaluación residían en un albergue para personas sin hogar. Los resultados mostraron que los patrones clínicos de personalidad y los síndromes clínicos de mayor prevalencia, de acuerdo al modelo de Millon, fueron: depresivo, narcisista y paranoide, así como la ansiedad, la drogodependencia y el trastorno del pensamiento, respectivamente. Además, el análisis de cluster clasificó a los sujetos en dos grupos «narcisista-adaptativo» $\mathrm{y}$ «depresivo-paranoide». Estos resultados sugieren que se deben adaptar los tratamientos y recursos sanitarios en función del deterioro psiquiátrico, considerando a las personas sin hogar como un colectivo heterogéneo con necesidades psicosociales diferentes.

Palabras clave: Sinhogarismo; persona sin hogar; trastornos de personalidad; perfil de personalidad.

\section{Introducción}

Se conoce como sinhogarismo al fenómeno social que se refiere a la falta prolongada de un hogar, de un marco estable y adecuado de convivencia para la persona (Avramov, 1995). En España no existen datos actualizados y exactos sobre cuántas personas están sin hogar.

Recibido: 25 de abril 2017; aceptado: 27 de septiembre 2017 Correspondencia: José Manuel Rodríguez-Pellejero, Universidad de Las Palmas de Gran Canaria, Departamento de Psicología y Sociología, Las Palmas de Gran Canaria, España. Email: jose. pellejero@ulpgc.es
Según el Instituto Nacional de Estadística (INE), en el año 2012 había en España al menos 22.938 personas sin hogar $(\mathrm{PsH})$ que se beneficiaban de algún servicio en centros asistenciales, 18.425 eran hombres y sólo 4.513 mujeres. El número total de PsH sería aún mayor, puesto que las personas que no solicitan ayudas sociales suelen quedar fuera de las Encuestas de Condiciones de Vida. En el año 2016, y según el INE la media de personas alojadas diariamente en albergues destinados a $\mathrm{PsH}$ fue de 16.347 , un $20,5 \%$ más que en el año 2014.

A pesar de ser un fenómeno de origen social, la falta de hogar está estrechamente relacionada con factores 
personales, subjetivos y psicológicos. El sinhogarismo, máxima expresión de la exclusión social, se ha relacionado con la enfermedad mental, el aumento de los costes sanitarios y la muerte prematura (Gozdzik, Salehi, O'Campo, Stergiopoulos, y Hwang, 2015; McCormick y White, 2016; Narendorf, 2017). Además, las PsH se hallan entre los colectivos con mayor riesgo de suicidio (Calvo-García, Giralt-Vázquez, Calvet-Roura, y Carbonells-Sánchez, 2016). Por su parte, el Manual Diagnóstico y Estadístico de los Trastornos Mentales, en su quinta edición, indica que se debe usar el código Z59.0 para las $\mathrm{PsH}$ en aquellos casos en los que: «la carencia de un hogar o residencia permanente tiene un impacto sobre el tratamiento o el pronóstico del individuo» (American Psychiatric Association, 2014, p.409).

Los estudios con PsH se han visto impulsados gracias al consenso alcanzado sobre el concepto de PsH. La Federación Europea de Organizaciones Nacionales y el Observatorio Europeo del Sinhogarismo, han desarrollado la categoría ETHOS (European Typology on Homelessness), una definición que es, a su vez, una clasificación (Busch-Geertsema, Culhane, y Fitzpatrick, 2016). En líneas generales esta clasificación coincide con el DSM-5, donde se considera que una persona no tiene hogar si su residencia nocturna principal es un refugio para gente sin hogar, un refugio temporal, un refugio contra la violencia doméstica, un espacio público, un edificio que no esté destinado a un uso residencial, una caja de cartón, una cueva o cualquier otra opción similar (American Psychiatric Association, 2014).

En España, las investigaciones realizadas para conocer las características y necesidades de las PsH son escasas y presentan grandes diferencias metodológicas, persistiendo el estereotipo de que las PsH son alcohólicos o enfermos mentales (Sánchez Morales, 2012). Aún así, la observación anterior no es incompatible con el hecho de que, cuando se realizan estudios comparativos entre población sin hogar y población general, la mayoría de las investigaciones señalaron una mayor incidencia de trastornos mentales en PSH, incluyendo trastornos de personalidad y drogodependencia (Dunne, Duggan, y O'Mahony, 2012; Edidin, Ganim, Hunter, y Karnik, 2012; Nishio et al., 2015; Salavera et al., 2014). En un estudio reciente con PsH se llegó a la conclusión de que el 86 \% de la muestra tenía un trastorno psiquiátrico diagnosticable, siendo el trastorno de la personalidad y el alcoholismo los más comunes en los varones y el trastorno de la personalidad y los trastornos de ansiedad en mujeres (Gazdag y Braun, 2015). En un metaanálisis anterior se seleccionaron veintinueve estudios que proporcionaban estimaciones obtenidas de una muestra de 5.684 PsH de siete países (Fazel, Khosla, Doll, y Geddes, 2008). Las conclusiones, de dicho metaanálisis, reportaron mayor prevalencia de trastornos de personalidad, enfermedad psicótica, depresión mayor, alcoholismo y drogodependencias. Como principal conclusión los autores señalan la necesidad de seguir profundizando en investigaciones sobre salud mental y PsH, con el fin de mejorar los modelos de atención psiquiátrica y social (Fazel et al., 2008).

El modelo de Millon reúne algunas características por las que resulta apropiado para la evaluación de la personalidad en PsH: incorpora los principios de la teoría evolutiva, supone una perspectiva integradora y resalta la importancia del continuo «normalidad-patología» (Cardenal, Sánchez, y Ortiz-Tallo, 2007). La perspectiva biosocial de Millon facilita la comprensión de la interacción entre las variables individuales y sociales. La personalidad es un factor principal en el origen de los diferentes síndromes clínicos y, al mismo tiempo, las relaciones sociales primarias son determinantes para el desarrollo de la personalidad (Millon, 1976; Millon y Everly, 1994). Además, este modelo cuenta con un instrumento de evaluación que permite la medición de sus constructos teóricos. El Inventario Clínico Multiaxial de Millon (MCMI-III) se ha mostrado como un instrumento fiable y válido en la población sin hogar (Stewart, 1998). Además, se ha utilizado para analizar patrones de personalidad y síndromes clínicos en muestras de PsH en España (Salavera et al., 2014; Salavera, Tricás, y Lucha, 2013).

Las PsH con trastornos mentales forman un grupo heterogéneo y caracterizado por múltiples comorbilidades (Fazel et al., 2008; Salavera, 2009). En población española, los estudios sobre personalidad y sinhogarismo se han centrado en analizar la frecuencia de los patrones clínicos de personalidad, resaltando también la alta prevalencia de trastornos de personalidad y drogodependencias, así como una frecuente comorbilidad entre los trastornos de personalidad, y de estos con otros trastornos mentales (Salavera, 2009; Salavera et al., 2014; Salavera, Puyuelo, Tricás, y Lucha, 2010; Salavera et al., 2013). A pesar de estas evidencias, las PsH suelen considerarse un grupo homogéneo, sesgo que dificulta el análisis de los factores que están relacionados con la falta de vivienda y con las diferentes patologías de esta población. Clark y Rich (2003) sugieren que la eficacia y el coste de los servicios para PsH se pueden mejorar si, en lugar de tratar a los enfermos mentales sin hogar como un grupo homogéneo, seleccionamos el tipo de servicio en función del deterioro psiquiátrico y del tipo de consumo de sustancias, otros estudios avalan esta indicación, sugiriendo que los programas de atención primaria de salud diseñados específicamente para $\mathrm{PsH}$ 
podrían ser más eficaces que la atención primaria de salud estándar (Hwang y Burns, 2014).

Los estudios tipológicos con PsH han demostrado que es posible hacer clasificaciones en función de diferentes patrones y sugieren que debemos seguir indagando en la caracterización de las PsH desde una perspectiva psicológica (Humphreys, 1995; Lee et al., 2016; Mavis, Humphreys, y Stöffelmayr, 1993; Morse, Calsyn, y Burger, 1992; Mowbray, Bybee, y Cohen, 1993; Solarz y Bogat, 1990; Stewart, 1998). Se han establecido tipologías según patrones de personalidad y síndromes clínicos en PsH utilizando el MCMI-III, concluyendo que el estudio tipológico favorece un conocimiento más profundo del sinhogarismo y puede facilitar la intervención, tanto desde los recursos sanitarios como desde los servicios sociales (Stewart, 1998).

En esta línea se sitúa nuestra investigación. Conviene advertir que no se trata de dar al sinhogarismo carácter de trastorno mental, sino de facilitar la adaptación de los tratamientos y recursos a un colectivo con necesidad de apoyos diferentes. Se establecen tres objetivos para este estudio ex post facto: (a) Analizar la prevalencia de patrones clínicos de la personalidad y síndromes clínicos en una muestra española de PsH en el municipio de Las Palmas de Gran Canaria; (b) explorar la relación entre patrones clínicos de la personalidad y síndromes clínicos en $\mathrm{PsH}$; (c) identificar perfiles de personalidad comunes en $\mathrm{PsH}$, e indagar en su relación con los síndromes clínicos.

\section{Método}

\section{Participantes}

Los participantes eran residentes de un centro de inclusión social para PsH. Se trata de un Centro de segunda estancia al cual se accede después de ser derivado desde otros Centros de baja exigencia para PsH, también desde Centros Municipales de Servicios Sociales y Comunidades terapéuticas de drogodependencia. Las condiciones que llevan a las personas a ser beneficiarios de este albergue son: ser una persona sin hogar de nacionalidad española, extranjero comunitario o con permiso de residencia, no estar en consumo activo de drogas o alcohol y manifestar la intensión de mantener la abstinencia durante el periodo de estancia. El primer criterio de participación en el estudio fue el de ser beneficiario de un albergue para PsH en el municipio. De esta manera, todos los participantes cumplían con la categoría ETHOS y la definición del DSM 5 para PsH. El segundo criterio de inclusión fue el de cronicidad en la situación de exclusión social grave. Se incluyeron en el estudio las personas que llevaban en situación de sin hogar más de un año, de las cuales el
$67 \%$ llevaba sin hogar un periodo de tiempo superior al año e inferior a tres, y el 33\% más de tres años. Se excluyeron a las personas que pudieran presentar una problemática de carácter exclusivamente económica y a los inmigrantes con menos de 5 años de residencia en España.

La muestra final estaba compuesta por 144 sujetos atendidos entre 2013 y 2015 en el Centro Residencial para PsH de Las Palmas de Gran Canaria, 35 de ellos eran mujeres y 109 hombres. El rango de edad estaba comprendido entre 19 y 63 años $(M=42.24 ; D T=$ 11.28).

\section{Instrumentos}

Con la finalidad de evaluar los patrones de personalidad y la prevalencia de síndromes clínicos se utilizó la adaptación española del MCMI-III (Cardenal et al., 2007). Es una escala de autoinforme que consta de 175 ítems con dos opciones de respuesta: verdadero o falso. Se evalúan 11 patrones de personalidad, más otros tres patrones considerados graves, y 10 síndromes clínicos. Cuenta además con cuatro escalas de validación del protocolo. Los patrones de personalidad evaluados son: esquizoide, evitativo, depresivo, dependiente, histriónico, narcisista, antisocial, agresivo-sádico, compulsivo, pasivo-agresivo y autodestructivo, además de los tres graves: esquizotípico, límite y paranoide. El MCMI-III considera que, para cada uno de los patrones, una puntuación típica entre 75 y 85 indica rasgos clínicos de personalidad, mientras que a partir de una puntuación de 85 indica un trastorno de personalidad. Los síndromes clínicos son: trastorno de ansiedad, trastorno somatomorfo, trastorno bipolar, trastorno distímico, dependencia del alcohol, dependencia de sustancias, trastorno de estrés postraumático, trastorno del pensamiento, depresión mayor y trastorno delirante. Las escalas de validación son: sinceridad validez, deseabilidad social devaluación.

En la validación española se obtuvieron consistencias internas entre .65 y .88 y una adecuada fiabilidad test-retest, entre .84 y .96 (Cardenal et al., 2007). En estudios posteriores el instrumento ha presentado una consistencia interna superior a .80 (Ortiz-Tallo, Cardenal, Ferragut, y Cerezo, 2011). En nuestra muestra, el análisis de consistencia interna mostró un valor alfa de .88 .

\section{Procedimiento}

Se trata de un estudio retrospectivo realizado a partir de los datos almacenados en la base de datos del Servicio Psicológico del Centro de acogida para PsH. De los 263 casos registrados en la base de datos durante el periodo comprendido entre los años 2013 y 2015, 119 
(45.24\%) no cumplían con los criterios de inclusión anteriormente señalados, o bien no permanecieron en el Centro el tiempo suficiente para que se les administrara el MCMI-III, quedando excluidos del estudio. Previamente, y en el resto de casos, el MCMI-III había sido administrado de forma individual por el psicólogo del Centro, una vez que la persona había adquirido la plaza de residente. La corrección se realizó por vía telemática. Antes de la evaluación se constataba que los participantes llevaban más de un mes de abstinencia de drogas, mediante analíticas de orina y alcohotest, eliminando así distorsiones por intoxicación o sintomatología abstinencial en las respuestas. En el momento de cumplimentar los cuestionarios, los participantes firmaban una hoja de consentimiento informado.

\section{Análisis estadístico de los datos}

Para evaluar la prevalencia de patrones clínicos de personalidad y síndromes clínicos, se calculó la frecuencia de los mismos, tomando como punto de corte la puntuación de tasa base $(\mathrm{TB})=75$, indicadora de patrón clínico de personalidad y de presencia de síndrome clínico (Cardenal et al., 2007). Con la finalidad de conocer la relación existente entre los patrones patológicos de personalidad de mayor frecuencia en esta población y los síndromes clínicos, se llevaron a cabo correlaciones bivariadas de Pearson.

El procedimiento utilizado para clasificar a los sujetos en perfiles comunes o grupos homogéneos fue el análisis de clusters sobre las puntuaciones en las 11 escalas de personalidad; este análisis se realizó en dos fases y a través del método jerárquico de Ward, donde la diferencia intergrupal se calcula mediante la distancia euclídea al cuadrado. Posteriormente, en el segundo análisis jerárquico, se forzó una solución con el número ideal de agrupaciones encontrado, clasificando así a todos los sujetos en alguna de ellas a partir de las puntuaciones en los patrones de personalidad. Por último, se analizaron las diferencias entre los perfiles comunes obtenidos en relación a los patrones graves de personalidad y a los síndromes clínicos, mediante una prueba $t$ para muestras independientes. Toda la información ha sido codificada y analizada mediante el programa estadístico SPSS ${ }^{\circledR} 24.0$ para Windows ${ }^{\circledR}$.

\section{Resultados}

\section{Análisis de frecuencias}

Los patrones clínicos de personalidad con una mayor frecuencia fueron el depresivo $(13.89 \%, n=20)$, narci- sista $(13.89 \%, n=20)$, paranoide $(13.19 \%, n=19)$, evitativo $(9.03 \%, n=13)$ y antisocial $(8.33 \%, n=12)$, mientras que el de menor prevalencia fue el agresivo, escala en la que ningún sujeto obtuvo una puntuación clínicamente significativa, considerando TB $>75$. Asimismo, los síndromes clínicos más frecuentes fueron la ansiedad $(58.33 \%, n=84)$, la dependencia de sustancias $(36.81 \%, n=53)$, el trastorno del pensamiento $(30.56 \%$, $n=44)$ y el trastorno bipolar $(21.53 \%, n=31)$. Los resultados se presentan en la Tabla 1.

Tabla 1. Patrones clínicos de la personalidad y síndromes clínicos. Frecuencias y porcentajes

\begin{tabular}{lrr}
\hline & $n$ & $\%$ \\
\hline Patología de la personalidad (TB $>$ 75) & & \\
Depresivo & 20 & 13.89 \\
Narcisista & 20 & 13.89 \\
Paranoide & 19 & 13.19 \\
Compulsivo & 14 & 9.72 \\
Evitativo & 13 & 9.03 \\
Antisocial & 12 & 8.33 \\
Esquizoide & 8 & 5.56 \\
Histriónico & 6 & 4.17 \\
Esquizotípico & 6 & 4.17 \\
Limite & 5 & 3.47 \\
Dependiente & 4 & 2.78 \\
Negativista (pasivo-agresivo) & 3 & 2.08 \\
Autodestructivo & 2 & 1.39 \\
Agresivo & 0 & 0.00 \\
Síndromes Clínicos (TB $>$ 75) & & \\
Ansiedad & 84 & 58.33 \\
Dependencia de sustancias & 53 & 36.81 \\
Trastorno del pensamiento & 44 & 30.56 \\
Bipolar & 31 & 21.53 \\
Trastorno delirante & 28 & 19.44 \\
Dependencia del alcohol & 27 & 18.75 \\
Depresión mayor & 25 & 17.36 \\
Estrés postraumático & 17 & 11.81 \\
Distimia & 16 & 11.11 \\
Trastorno somatomorfo & 13 & 9.03 \\
\hline & &
\end{tabular}

\section{Análisis de correlaciones}

Con la finalidad de examinar la relación entre patrones clínicos de la personalidad y síndromes clínicos en PsH se realizó un análisis de correlaciones. En la Tabla 2 se muestran los resultados de las relaciones entre los 6 patrones clínicos de personalidad con mayor prevalencia en la muestra de PsH y los síndromes clínicos analizados. Los resultados muestran que el patrón de personalidad depresivo se relaciona de forma positiva y significativa con todos los síndromes clínicos excepto con el abuso de drogas y de alcohol. El patrón narcisista corre- 
Tabla 2. Coeficientes de correlación entre patrones clínicos de personalidad y síndromes clínicos

\begin{tabular}{|c|c|c|c|c|c|c|c|c|c|c|c|c|c|c|c|}
\hline & 1 & 2 & 3 & 4 & 5 & 6 & 7 & 8 & 9 & 10 & 11 & 12 & 13 & 14 & 15 \\
\hline \multicolumn{16}{|l|}{ 1. Depresivo } \\
\hline 2. Narcisista & $-.41 * *$ & & & & & & & & & & & & & & \\
\hline 3. Paranoide & $.36^{* *}$ & -.07 & & & & & & & & & & & & & \\
\hline 4. Compulsivo & $-.30 * *$ & $.21 *$ & $-.22 * *$ & & & & & & & & & & & & \\
\hline 5. Evitativo & $.48^{* *}$ & $-.60 * *$ & $.34 * *$ & $-.31 * *$ & & & & & & & & & & & \\
\hline 6.Antisocial & .10 & .04 & .01 & $-.56^{* *}$ & -.09 & & & & & & & & & & \\
\hline 7. Ansiedad & $.66^{* *}$ & $-.38 * *$ & $.49^{* *}$ & $-.32 * *$ & $.45^{* *}$ & .16 & & & & & & & & & \\
\hline 8. Abuso sustancias & .12 & -.08 & .06 & $-.46 * *$ & -.04 & $.75^{* *}$ & $.20^{*}$ & & & & & & & & \\
\hline 9. Abuso de alcohol & .13 & -.04 & $.20^{*}$ & $-.44 * *$ & .03 & $.54 * *$ & $.21^{*}$ & $.48^{* *}$ & & & & & & & \\
\hline 10. Tr. Pensamiento & $.65^{* *}$ & $-.38 * *$ & $.52 * *$ & $-.49 * *$ & $.50^{* *}$ & $.17 *$ & $.77^{* *}$ & $.18^{*}$ & $.26^{* *}$ & & & & & & \\
\hline 11. Bipolar & $.23^{* *}$ & .01 & $.36^{* *}$ & $-.28 * *$ & .04 & $.18^{*}$ & $.37 * *$ & .12 & $.31^{* *}$ & $.40 * *$ & & & & & \\
\hline 12. Delirante & $.24 * *$ & .15 & $.71^{* *}$ & -.10 & 0.1 & -.02 & $.37 * *$ & .05 & .10 & $.36^{* *}$ & $.34^{* *}$ & & & & \\
\hline 13. Depresión mayor & $.56^{* *}$ & $-.29 * *$ & $.47^{* *}$ & $-.36^{*}$ & $.37^{* *}$ & .08 & $.65^{* *}$ & .08 & .16 & $.79 * *$ & $.25^{* *}$ & $.36^{* *}$ & & & \\
\hline 14. Estrés Postraumático & $.71^{* *}$ & $-.43 * *$ & $.50^{* *}$ & $-.33 * *$ & $.47^{* *}$ & .10 & $.82^{* *}$ & .12 & $.20^{*}$ & $.79 * *$ & $.32^{* *}$ & $.31^{* *}$ & $.73^{* *}$ & & \\
\hline 15. Distimia & $.70^{* *}$ & $-.38 * *$ & $.48^{* *}$ & $-.39 * *$ & $.46^{* *}$ & .08 & $.62^{* *}$ & .12 & $.27^{* *}$ & $.73^{* *}$ & $.31^{* *}$ & $.37 * *$ & $.75^{* *}$ & $.70^{* *}$ & \\
\hline 16. Somatomorfo & $.53^{* *}$ & $-.29 * *$ & $.51^{* *}$ & $-.29 * *$ & $.38^{* *}$ & .01 & $.60^{* *}$ & .10 & .12 & $.67 * *$ & $.28^{* *}$ & $.40 * *$ & $.86^{* *}$ & $.64^{* *}$ & $.70^{* *}$ \\
\hline
\end{tabular}

Nota: ${ }^{*} p<.05 ; * *<.01$.

laciona de forma negativa y significativa $(p<.01)$ con la ansiedad, el trastorno del pensamiento, la depresión mayor, el estrés postraumático, la distimia y los trastornos somatomorfos. El patrón paranoide también se asocia positiva y significativamente $(p<.05)$ con todos los síndromes clínicos, excepto con el abuso de drogas. Además, el patrón de personalidad compulsivo correlaciona negativamente con todos y cada uno de los síndromes clínicos, sin excepción. Por su parte, el patrón de personalidad evitativo correlaciona de forma positiva con todos los síndromes clínicos excepto con el abuso de drogas, cuya relación es negativa y no significativa $(p>$ .05 ). De forma similar, el patrón de personalidad antisocial se relaciona de forma positiva con todos los síndromes clínicos excepto con el trastorno delirante que lo hace de forma negativa y no significativa $(p>.05)$.

\section{Análisis de clúster y de medias entre grupos}

Con el objetivo de identificar perfiles de personalidad comunes en PsH se llevó a cabo un primer análisis de clúster con carácter exploratorio para las 11 escalas de personalidad, utilizando el total de la muestra. En el historial de conglomeración del primer análisis jerárquico encontramos el aumento más importante de coeficientes cuando se pasa de dos conglomerados $(\mathrm{C}=358295.19)$ a uno $(\mathrm{C}=535128.92)$, siendo el aumento de 176833.73. Tomando como base la lectura del dendograma y los resultados lógicos que se obtuvieron, se seleccionó una solución de dos grupos como el número más adecuado de cluster. Posteriormente, se realizó un nuevo análisis jerárquico forzando la solución única de dos clúster. Al grupo 1 se asignaron 60 sujetos (41.67\%); este grupo se etiqueta como grupo «narcisista-adaptativo», según las puntuaciones más altas en las escalas de personalidad. Al grupo 2 se asignaron 84 sujetos (58.33\%), al que se denomina grupo «depresivo-paranoide».

En la Tabla 3 se presentan los resultados obtenidos por cada grupo en el MCMI-III, tanto en las escalas de personalidad como en los síndromes clínicos, y las diferencias significativas entre ambos grupos en las escalas de dicho cuestionario.

El grupo 1 se caracteriza por obtener puntuaciones altas y significativamente superiores en las escalas narcisista, compulsiva e histriónica. El grupo 2 destaca por unas puntuaciones muy elevadas y significativamente superiores en las escalas de personalidad depresiva, paranoide, evitativa y límite, así como por tener puntuaciones más altas en la mayoría de los síndromes clínicos. En general, la totalidad de la muestra evidenció altas puntuaciones en las escalas agresividad y antisocial, sin que se hallaran diferencias significativas entre los grupos para dichos rasgos.

Respecto a los síndromes clínicos, el grupo depresivo-paranoide evidenció puntuaciones significativamente superiores en todas las escalas consideradas de mayor gravedad, con especial relevancia en los trastornos de ansiedad, trastorno del pensamiento, trastorno bipolar y distimia. No existen diferencias significativas entre los grupos en lo referente a abuso de sustancias y dependencia del alcohol, siendo las medias cercanas y elevadas para ambos grupos. 
Tabla 3. Puntuaciones de cada grupo en las escalas de personalidad, en los síndromes clínicos y valor del estadístico t-Student

\begin{tabular}{|c|c|c|c|c|c|}
\hline & \multicolumn{2}{|c|}{ Grupo $1(n=60)$} & \multicolumn{3}{|c|}{ Grupo $2(n=84)$} \\
\hline & $M$ & $D T$ & $M$ & $D T$ & $t$ \\
\hline \multicolumn{6}{|l|}{ Escalas de personalidad } \\
\hline Narcisista & 71.45 & 10.37 & 52.02 & 16.50 & $8.04 * * *$ \\
\hline Compulsivo & 59.60 & 16.64 & 42.40 & 17.79 & $5.87 * * *$ \\
\hline Antisocial & 59.02 & 16.77 & 60.75 & 14.92 & -.65 \\
\hline Agresivo & 54.95 & 16.14 & 56.94 & 8.76 & -.95 \\
\hline Paranoide & 54.92 & 22.24 & 68.31 & 13.43 & $-4.49 * * *$ \\
\hline Histrionico & 53.17 & 14.47 & 26.06 & 16.59 & $10.18 * * *$ \\
\hline Negativista & 50.28 & 19.90 & 58.93 & 11.45 & $-3.29 * *$ \\
\hline Esquizoide & 48.70 & 18.83 & 61.56 & 10.98 & $-5.15 * * *$ \\
\hline Limite & 45.88 & 20.75 & 62.40 & 9.80 & $-6.37 * * *$ \\
\hline Depresivo & 41.83 & 24.48 & 67.98 & 10.14 & $-8.79 * * *$ \\
\hline Autodestructivo & 38.85 & 21.31 & 57.42 & 7.44 & $-7.38 * * *$ \\
\hline Esquizotipico & 37.98 & 23.52 & 61.94 & 15.16 & $-7.42 * * *$ \\
\hline Evitativo & 29.75 & 15.88 & 65.42 & 11.08 & $-15.87 * * *$ \\
\hline Dependiente & 24.90 & 17.11 & 58.02 & 11.97 & $-13.67 * * *$ \\
\hline \multicolumn{6}{|l|}{ Síndromes clínicos } \\
\hline Ansiedad & 52.92 & 33.47 & 84.00 & 18.38 & $-7.14 * * *$ \\
\hline Trastorno del pens. & 39.37 & 25.36 & 72.62 & 20.17 & $-8.75 * * *$ \\
\hline Abuso sustancias & 65.68 & 22.08 & 68.12 & 20.63 & -.68 \\
\hline Bipolar & 58.48 & 17.11 & 66.81 & 16.82 & $-2.90 *$ \\
\hline Distimia & 39.37 & 25.00 & 66.40 & 15.14 & $-8.06 * * *$ \\
\hline Trastorno delirante & 59.57 & 24.75 & 66.02 & 19.69 & -1.74 \\
\hline Estrés post. & 39.25 & 25.87 & 65.86 & 19.92 & $-6.97 * *$ \\
\hline Abuso de alcohol & 58.60 & 21.34 & 64.55 & 16.23 & -1.89 \\
\hline Depresión mayor & 37.22 & 28.55 & 63.67 & 23.67 & $-6.06 * * *$ \\
\hline Trastorno somat. & 34.17 & 26.70 & 58.44 & 21.49 & $-6.03 * * *$ \\
\hline
\end{tabular}

Nota: $* p<.05 ; * * p<.01 ; * * * p<.001$.

\section{Discusión}

Ante la necesidad de adaptar la intervención con PsH a sus patrones de personalidad, este trabajo plantea tres objetivos: analizar la prevalencia de patrones clínicos de personalidad y síndromes clínicos en una muestra de PsH del municipio de Las Palmas de Gran Canaria, examinar la relación entre síndromes clínicos y patrones de personalidad patológicos y, finalmente, identificar perfiles de personalidad comunes en PsH.

En relación al primer objetivo propuesto, los patrones clínicos de personalidad mostraron alta prevalencia en la población estudiada, en concordancia con otros estudios de PsH (Fazel et al., 2008; Narendorf, 2017). Los de mayor prevalencia fueron: depresivo, narcisista, paranoide, compulsivo, evitativo y antisocial, por este orden. Estos resultados están en consonancia con estudios anteriores realizados con población española de PsH en los que también se halló una elevada frecuencia de los patrones evitativos, compulsivos, antisociales y narcisistas (Salavera, 2009; Salavera et al., 2014; Salavera et al.,
2013). A diferencia de los estudios anteriores, los resultados del presente trabajo ofrecen porcentajes de prevalencia menores, salvo en los patrones esquizoide y dependiente, donde los porcentajes encontrados son mayores. Estas diferencias pueden explicarse a partir del tamaño de la muestra, el punto de corte y las versiones del cuestionario que se han utilizado. En los estudio anteriores la muestra utilizada fue menor y, de acuerdo, con las conclusiones de Fazel et al. (2008), las menores tasas de participación se asocian con mayores prevalencias en trastornos de personalidad. Por otro lado, los estudios de Salavera toman una tasa base de 74 como punto de corte, menor que la tasa de 75 que utilizamos en nuestro caso, además sus investigaciones se han llevado a cabo con la segunda versión del cuestionario de Millon (MCMI-II), versión que no incluía aún la escala de personalidad depresiva.

Otras investigaciones han utilizaron el MCMI-III con una amplia muestra española que padecía diferentes trastornos clínicos, pero sin problemas de sinhogarismo (Ortiz-Tallo et al., 2011). Estos autores encontraron que 
los patrones de personalidad de mayor prevalencia fueron: compulsiva, narcisista e histriónica. Si comparamos esos resultados con los de esta investigación, observamos que los patrones depresivo, paranoide, evitativo y antisocial emergen como característicos del grupo de $\mathrm{PsH}$. Los patrones depresivos pueden entenderse porque, tener una visión negativa del presente y del futuro es algo natural para las personas que han visto cronificada su situación de PsH, esta visión negativa se retroalimenta socialmente, aumentando la probabilidad de que la persona integre una autoestima baja y sentimientos de impotencia, precisamente lo que evalúa la escala de personalidad depresiva del MCMI-III. La baja autoestima de las PsH se puede proyectar también mediante la ecuación narcisista de: «yo soy diferente y por lo tanto superior». Las altas puntuaciones en patrones paranoides pueden estar determinadas por la sensación amenaza-peligro-agresión que experimentan las PsH. En circunstancias de desprotección, la suspicacia y la desconfianza hacia los demás son estrategias de afrontamiento reforzadas por el contexto. Los problemas de auto-aceptación y la falta de propósito vital pueden explicar las puntuaciones en la escala de personalidad evitativa, ya que las estrategias de afrontamiento evitativas predicen este tipo de problemas (Sanjuán y Ávila, 2016). Se especula con la idea de que la falta de flexibilidad característica de la personalidad paranoide y narcisista, a la que se suma el retraimiento de la personalidad evitativa, pueden estar en el origen, y ser a la vez consecuencia, de la ruptura de lazos sociales, principal característica de las PsH. Las altas puntuaciones en rasgos antisociales pueden guardar relación con la conflictividad social propia de los contextos de marginación, con el incumplimiento de normas y los problemas con la ley.

Respecto a los síndromes clínicos, los resultados de nuestro estudio sugieren una alta incidencia de estos en $\mathrm{PsH}$, apoyando los resultados encontrados en estudios longitudinales realizados también con PsH (Hodgson, Shelton y Bree, 2015). Los síndromes clínicos de mayor prevalencia en nuestra muestra son: la ansiedad, la drogodependencia, el trastorno del pensamiento, los trastornos del estado de ánimo y, en menor medida, el trastorno delirante, el alcoholismo y el estrés postraumático. En concreto, Fazel et al. (2008) concluyeron en su metanálisis que la mayoría de los estudios habían reportado una mayor prevalencia en trastornos de personalidad, trastornos psicóticos, alcoholismo y drogodependencia, respecto a la población «normal». Al comparar nuestros resultados y los de investigaciones similares, en cuanto a población e instrumentos de evaluación, encontramos similitudes en la alta prevalencia de drogodependencia, trastorno delirante y trastorno del pensamiento (Salave- ra, 2009; Salavera et al., 2010; Stewart, 1998). Estos resultados se pueden explicar por el hecho de que las PsH están sometidas a múltiples estresores, pudiendo presentar además fobia social, circunstancias que aumentan la ansiedad. La drogodependencia está relacionada con conflictividad social, puede ser causa y efecto de los procesos de exclusión social. Las altas puntuaciones en la escala de trastorno del pensamiento en PsH no indican necesariamente sintomatología psicótica, es probable que se deba a que esta dimensión evalúa aspectos como: percepción de ser incomprendido, de estar aislado, pensamiento autorreferencial, conductas retraídas, solitarias y secretas. Estos comportamientos, que en una persona integrada resultan extraños, en una PsH adquieren sentido por sí mismos.

En cuanto al segundo de los objetivos planteados, se obtuvieron correlaciones significativas entre los patrones clínicos de personalidad y todos los síndromes clínicos del MCMI-III, excepto para el abuso de alcohol y drogas. Conviene señalar que las relaciones entre los patrones narcisista, histriónico y compulsivo, fueron en sentido negativo, mientras que las relaciones del resto de patrones de personalidad con los síndromes clínicos fueron de signo positivo. Estos resultados son congruentes con los hallados por los autores del MCMI-III e investigaciones posteriores, que advirtieron de que las escalas de personalidad histriónica, narcisista y compulsiva, correlacionaban negativamente con los síndromes clínicos, porque tienden a incluir rasgos normales (Boyle y Le Déan, 2000; Cardenal et al., 2007; Craig, 2005; Millon, 1976; Millon y Everly, 1994; Ortiz-Tallo et al., 2011). Las altas puntuaciones en dependencia de alcohol y otras sustancias parecen indicar que alcoholismo y la drogodependencia son problemas comunes en $\mathrm{PsH}$, apoyando los hallazgos de otros estudios realizados con PsH (Fazel et al., 2008; Salavera et al., 2010; Stewart, 1998), y los de las investigaciones que relacionaron la personalidad antisocial y narcisista con el consumo de drogas (Craig y Olson, 1990; Fals-Stewart, 1992; Retzlaff y Gibertini, 1990).

Para la consecución del tercer objetivo propuesto, se realizó un análisis de cluster a partir de las escalas de personalidad del MCMI-III. Los resultados identificaron dos grupos preponderantes. El primero se denominó «narcisista-adaptativo». Los individuos de este grupo se caracterizan por la falta de reconocimiento y su necesidad de «gustar», tienden a no admitir fácilmente problemas de salud mental y, aún así, presenta mayor ajuste personal y mejores estrategias de afrontamiento que los individuos del segundo cluster. Este grupo coincide parcialmente con uno de los subgrupos que Stewart identificó en una población de PsH de los Estados Unidos, al 
que denominó «Negadores» (Stewart, 1998), advirtiendo que puede parecer que tienen sólo problemas económicos y sociales, pero que en realidad se trata de individuos problemáticos en los albergues para PsH. Así, más que un grupo con «buen funcionamiento», como lo han llamado en investigaciones similares (Humphreys, 1995; Kahn, Hannah, Hinkin, Montgomery, y Pitz, 1987; Mavis et al., 1993; Morse et al., 1992; Mowbray et al., 1993; Solarz y Bogat, 1990), es un grupo negador de sus problemas de salud mental o drogodependencia (Stewart, 1998).

El segundo cluster que hemos aislado se denomina «depresivo-paranoide»; los individuos de este grupo tienden a la melancolía, al pesimismo, al resentimiento, a mostrar actitudes de desconfianza, suspicacia y sentimientos de desconexión. Este grupo puntúa más alto en la mayoría de los síndromes clínicos y trastornos de personalidad severos, evidenciando cierta huella de padecer acumulación de traumas o traumas no resueltos, en congruencia con los resultados de otros estudios con PsH (Lippert y Lee, 2015; Torchalla, Li, Strehlau, Linden, y Krausz, 2014). Algunos estudios realizados en muestras españolas, pero sin problemas de sinhogarismo, han relacionado también la psicopatología emocional con los distintos rasgos de personalidad, señalando dos rasgos de la personalidad como relevantes para los trastornos del estado de ánimo: la Emocionalidad y la Extraversión (Roncero, Fornés, García-Soriano y Belloch, 2014). En concordancia con estos hallazgos, la alta emocionalidad y la baja extraversión son también rasgos de personalidad del grupo denominado «depresivo-paranoide», el grupo con más problemas del estado de ánimo. Además, el grupo «depresivo-paranoide» coincide en gran medida con el grupo que Stewart (1998) y otros autores llamaron multiproblema (Humphreys, 1995; Kahn et al., 1987; Mavis et al., 1993; Morse et al., 1992; Solarz y Bogat, 1990). Se trata de un grupo de difícil adaptabilidad, variable individual que ha sido relacionada con el mayor riesgo de suicidio en población general, y con el abandono temprano de los procesos de incorporación social en PsH (Salavera et al., 2013; Stewart,1998).

La clasificación tipológica a la que se ha llegado en este estudio es similar a la obtenida en la investigación realizada por Stewart (1998). Las diferencias entre grupos en cuanto a síndromes clínicos se han mantenido según lo esperado y en coherencia con otros estudios realizados con PsH (Humphreys, 1995; Kahn et al., 1987; Mavis et al., 1993; Morse et al., 1992; Mowbray et al., 1993; Solarz y Bogat, 1990;). Sin embargo, los resultados no han permitido replicar la presencia de los subtipos; «abuso de sustancias»o «antisocial», variables con altas puntuaciones en los dos cluster encontrados. Es posible que esto se deba a la cronificación del sinhogarismo en la muestra estudiada, puesto que la variable abuso de sustancias tiende a aumentar en función del tiempo que lleva el individuo sin hogar (Panadero-Herrero y Muñoz-López, 2014), y en la muestra estudiada la cronificación del sinhogarismo fue uno de los criterios de inclusión. En síntesis, el grupo depresivo-paranoide tiene peor pronóstico que el narcisista-adaptativo, presentan mayor sintomatología clínica en general y mayor índice de abandono de programas. El alcoholismo y la drogodependencia aparecen como principales síndromes clínicos del sinhogarismo, con independencia de los perfiles de personalidad.

Estos hallazgos pueden resultar de utilidad a varios niveles. Se contribuyó a identificar las características de personalidad que pueden estar funcionando como un factor más de exclusión social. Las descripciones de los estilos de personalidad que se ofrecen pueden ayudar en el diseño de programas de intervención cognitivo-conductual diferenciados para cada perfil. Por ejemplo, con directrices sólidas y límites firmes para el grupo narcisista-adaptativo, frente a pautas flexibles que favorezcan una relación de colaboración para el grupo depresivo-paranoide. Mientras que los objetivos terapéuticos para el primer grupo se pueden centrar en aumentar la responsabilidad conductual, el comportamiento de reciprocidad, la sensibilidad hacia los sentimientos del Otro, etc. Los programas destinados al grupo depresivo-paranoide pueden orientar sus objetivos hacia la reducción de la sensibilidad a las críticas externas y el aumento progresivo de la sensación de autoeficacia. Este último grupo requiere mayor entrenamiento en habilidades interpersonales, mejorar su regulación emocional y el manejo de la ansiedad, por esto, pueden llegar a beneficiarse de terapias como la dialéctica conductual de Linehan (2003) u otras similares. El conocimiento del estilo de personalidad de las PsH puede ser de utilidad también a trabajadores y educadores sociales, para mejorar las técnicas de acompañamiento, así como para realizar pronósticos sobre abandono anticipado del proceso de inclusión. Finalmente, los resultados de este estudio pueden contribuir a mejorar las predicciones del riesgo real de suicidio en $\mathrm{PsH}$.

El estudio presenta algunas limitaciones. Por un lado, la selección de la muestra se encuentra restringida y localizada en albergues de un solo municipio, lo que limita la generalización de los resultados. Por otro lado, aunque la distribución de género de la muestra es la habitual en los albergues para PsH, la escasa presencia de mujeres puede haber limitado la fiabilidad de los resultados. No se han establecido diferencias en función del tiempo sin hogar, la edad y el sexo, ni de los motivos que lleva- 
ron al sinhogarismo. Al tratarse de un estudio de carácter transversal, no se puede conocer si los trastornos favorecen el sinhogarismo o si el sinhogarismo contribuye a la aparición o consolidación de síndromes clínicos; estudios prospectivos o longitudinales pueden arrojar luz sobre esta cuestión. También sería pertinente la validación adicional de los resultados del MCMI-III mediante entrevista clínica. Una línea de intervención futura es el estudio del poder predictivo que pueden tener estas tipologías de personalidad sobre los índices de suicidio de las PsH, así como sobre su prevención.

En conclusión, los resultados mostraron que existe relación entre patrones clínicos de personalidad y síndromes clínicos en $\mathrm{PsH}$, con una mayor prevalencia en los patrones depresivo, narcisista y paranoide, en cuanto a trastornos de personalidad, y en la ansiedad, la drogodependencia y el trastorno del pensamiento, en cuanto a los síndromes clínicos. Además, se obtuvieron dos perfiles de personalidad, «narcisista-adaptativo» y «depresivo-paranoide», con diferencias significativas y pronóstico diferenciado. Estos resultados indican la conveniencia de adaptar los tratamientos y recursos sanitarios en función del patrón de personalidad que presenten las $\mathrm{PsH}$.

\section{Conflicto de intereses}

Los autores declaran que no existen conflictos de intereses.

\section{Referencias}

Asociación Americana de Psiquiatría (2014). Manual diagnóstico y estadístico de los trastornos mentales (DSM-5), $5^{\text {a }}$ ed. Arlington, VA: Asociación Americana de Psiquiatría.

Avramov, D. (1995). Homelessness in the European Union: Social and legal context of housing exclusion in the 1990s: Fourth research report of the European observatory on homelessness. Bruselas: Feantsa.

Boyle, G. J., \& Le Déan, L. (2000). Discriminant validity of the illness behavior questionnaire and Millon Clinical Multiaxial Inventory III in a heterogeneous sample of psychiatric outpatients. Journal of Clinical Psychology, 56, 779-791. doi: 10.1002/(SICI)1097-4679(200006)56:6<779::AID-JCL$\mathrm{P} 7>3.0 . \mathrm{CO} ; 2-7$

Busch-Geertsema, V., Culhane, D., \& Fitzpatrick, S. (2016). Developing a global framework for conceptualizing and measuring homelessness. Habitat International, 55, 124-132. doi:10.1016/j.habitatint.2016.03.004

Calvo-García, F., Giralt-Vázquez, C., Calvet-Roura, A., y Carbonells-Sánchez, X. (2016). Riesgo de suicidio en población sin hogar. Clínica y Salud, 27, 89-96. doi:10.1016/j.clysa.2016.05.002

Cardenal, V., Sánchez, M. P., y Ortiz-Tallo, M. (2007). Adaptación y baremación al Español del Inventario Clínico Multiaxial de Millon-III (MCMI-III). Madrid: TEA, Ediciones.
Clark, C., \& Rich, A. R. (2003). Outcomes of homeless adults with mental illness in a housing program and in case management only. Psychiatric Services, 54, 78-83. doi: 10.1176/appi. ps.54.1.78

Craig, R. J. (2005). New directions in interpreting the Millon Clinical Multiaxial Inventory-III. New York, NY: John Wiley \& Sons.

Craig, R. J., \& Olson, R. E. (1990). MCMI comparisons of cocaine abusers and heroin addicts. Journal of Clinical Psychology, 46, 230-237. doi. 10.1002/1097-4679(199003)46:2<230:: AID-JCLP2270460217>3.0.CO;2-7

Dunne, E., Duggan, M., \& O'Mahony, J. (2012). Mental health services for homeless: Patient profile and factors associated with suicide and homicide. Irish Medical Journal, 105(3), 7174.

Edidin, J. P., Ganim, Z., Hunter, S. J., \& Karnik, N. S. (2012). The mental and physical health of homeless youth: A literature review. Child Psychiatry \& Human Development, 43, 354-375. doi: 10.1007/s10578-011-0270-1

Fals-Stewart, W. (1992). Personality characteristics of substance abusers: An MCMI cluster typology of recreational drug users treated in a therapeutic community and its relationship to length of stay and outcome. Journal of Personality Assessment, 59, 515-527. doi:10.1207/s15327752jpa5903_7

Fazel, S., Khosla, V., Doll, H., \& Geddes, J. (2008). The prevalence of mental disorders among the homeless in western countries: Systematic review and meta-regression analysis. PLoS Medicine, 5(12), e225. doi:10.1371/journal.pmed.0050225

Gazdag, G., \& Braun, E. (2015). Prevalence of psychiatric disorders in a homeless population. Journal of Psychosomatic Research, 78, 600-601. doi: 10.1016/j.jpsychores.2015.03.051

Gozdzik, A., Salehi, R., O'Campo, P., Stergiopoulos, V., \& Hwang, S. W. (2015). Cardiovascular risk factors and 30-year cardiovascular risk in homeless adults with mental illness. $B M C P u$ blic Health, 15, 165. doi:10.1186/s12889-015-1472-4

Hodgson, K. J., Shelton, K. H., \& Bree, M. (2015). Psychopathology among young homeless people: longitudinal mental health outcomes for different subgroups. British Journal of Clinical Psychology, 54, 307-325. doi:10.1111/bjc.12075

Humphreys, K. (1995). Sequential validation of cluster analytic subtypes of homeless veterans. American Journal of Community Psychology, 23, 75-98. doi:10.1007/BF02506923

Hwang, S. W., \& Burns, T. (2014). Health interventions for people who are homeless. The Lancet, 384, 1541-1547. doi:10.1016/ S0140-6736(14)61133-8

Kahn, M. W., Hannah, M., Hinkin, C., Montgomery, C., \& Pitz, D. (1987). Psychopathology on the streets: Psychological assessment of the homeless. Professional Psychology: Research and Practice, 18, 580-586. doi:10.1037/0735-7028.18.6.580

Lee, C. T., Guzman, D., Ponath, C., Tieu, L., Riley, E., \& Kushel, M. (2016). Residential patterns in older homeless adults: Results of a cluster analysis. Social Science \& Medicine, 153, 131-140. doi:10.1016/j.socscimed.2016.02.004

Lineham, M. M. (2003). Manual de tratamiento de los trastornos de personalidad límite. Barcelona: Paidós.

Lippert, A. M., \& Lee, B. A. (2015). Stress, coping, and mental health differences among homeless people. Sociological Inquiry, 85, 343-374. doi:10.1111/soin. 12080 
Mavis, B. E., Humphreys, K., \& Stöffelmayr, B. E. (1993). Treatment needs and outcomes of two subtypes of homeless persons who abuse substances. Hospital \& Community Psychiatry, 44, 1185-1187.

McCormick, B., \& White, J. (2016). Hospital care and costs for homeless people. Clinical Medicine, 16, 506-510. doi: 10.7861/clinmedicine.16-6-506

Millon, T. (1976). Psicopatología moderna: Enfoque biosocial de los aprendizajes erróneos y de los disfuncionalismos. Barcelona: Salvat.

Millon, T., \& Everly, G. S. (1994). La personalidad y sus trastornos. Barcelona: Martínez Roca.

Morse, G. A., Calsyn, R. J., \& Burger, G. K. (1992). Development and cross-validation of a system for classifying homeless persons. Journal of Community Psychology, 20, 228-242. doi: $10.1002 / 1520-6629(199207) 20: 3<228$ ::AID-JCOP2290200306>3.0.CO;2-0

Mowbray, C. T., Bybee, D., \& Cohen, E. (1993). Describing the homeless mentally ill: Cluster analysis results. American Journal of Community Psychology, 21, 67-93. doi: 10.1007/ BF00938208

Narendorf, S. C. (2017). Intersection of homelessness and mental health: A mixed methods study of young adults who accessed psychiatric emergency services. Children and Youth Services Review, 81, 54-62. doi: 10.1016/j.childyouth.2017.07.024

Nishio, A., Yamamoto, M., Horita, R., Sado, T., Ueki, H., Watanabe, T., ... Shioiri, T. (2015). Prevalence of mental illness, cognitive disability, and their overlap among the homeless in Nagoya, Japan. PLoS: e0138052. doi:10.1371/journal.pone. 0138052

Ortiz-Tallo, M., Cardenal, V., Ferragut, M., y Cerezo, M. V. (2011). Personalidad y síndromes clínicos: un estudio con el MCMI-III basado en una muestra española. Revista de Psicopatología y Psicología Clínica, 16, 49-59. doi: 10.5944/rppc. vol.16.num.1.2011.10350

Panadero-Herrero, S., \& Muñoz-López, M. (2014). Salud, calidad de vida y consumo de sustancias en función del tiempo en situación sin hogar. Anales de Psicología, 30, 70-77. doi:10.6018/analesps.30.1.137911

Retzlaff, P. D., \& Gibertini, M. (1990). Factor-based special scales for the MCMI. Journal of Clinical Psychology, 46, 47-52. doi: AID-JCLP2270460108>3.0.CO;2-J
Roncero, M., Fornés, G., García-Soriano, G., y Belloch, A. (2014). Modelo de personalidad HEXACO: Relaciones con psicopatología emocional en una muestra española. Revista de Psicopatología y Psicología Clínica, 19, 1-14. doi: 10.5944/rppc. vol.19.num.1.2014.12929

Salavera, C. (2009). Personality disorders in homeless people. International Journal of Psychology and Psychological Therapy, 9, 275-283. doi: 10.5460/jbhsi.v1.1.380

Salavera, C., Antoñanzas, J. L., Bustamante, J. C., Carrón, J., Usán, P., Teruel, P., . . Cerra, R. (2014). Comorbidity of attention deficit hyperactivity disorder with personality disorders in homeless people. BMC Research Notes, 7, 916. doi:10.1186/1756-0500-7-916

Salavera, C., Puyuelo, M., Tricás, J.M., \& Lucha, O. (2010). Comorbilidad de trastornos de personalidad: estudio en personas sin hogar. Universitas Psychologica, 9, 471-481.

Salavera, C., Tricás, J. M., \& Lucha, O. (2013). Personality disorders and treatment drop out in the homeless. Neuropsychiatric Disease and Treatment, 9, 379-387. doi:10.2147/NDT.S38677

Sánchez Morales, M. R. (2012). En los límites de la exclusión social: Las personas sin hogar en España. OBETS. Revista de Ciencias Sociales, 7, 307-324. doi: 10.14198/OBETS2012. 7.2.06

Sanjuán, P., y Avila, M. (2016). Afrontamiento y motivación como predictores del bienestar subjetivo y psicológico. Revista de Psicopatología y Psicología Clínica, 21(1), 1-10. doi: 10.5944/rppc.vol.21.num.1.2016.15401

Solarz, A., \& Bogat, G. A. (1990). When social support fails: The homeless. Journal of Community Psychology, 18, 79-96. doi: $10.1002 / 1520-6629$ (199001) $18: 1<79:$ : A ID-JCOP2290180112>3.0.CO;2-B

Stewart, K. S. (1998). A study of a homeless population through the use of the MCMI-III (Order No. 9841816). Available from ProQuest Dissertations \& Theses Global. (304482325). Retrieved from https://search.proquest.com/docview/30448 2325 ? accountid $=14705$

Torchalla, I., Li, K., Strehlau, V., Linden, I. A., \& Krausz, M. (2014). Religious participation and substance use behaviors in a Canadian sample of homeless people. Community Mental Health Journal, 50, 862-869. doi: 10.1007/s10597-0149705-z 